\title{
Pathology of the Central Autonomic Nervous System in Stillbirth
}

\author{
Luigi Matturri* and Anna Maria Lavezzi
}

\author{
“Lino Rossi" Research Center, Institute of Pathology, University of Milan, Italy
}

\begin{abstract}
The aim of this study was to identify in stillbirth a possible involvement of morphological and/or physiological alterations of structures of the central autonomic nervous system in the mechanism of death. The study, including the indepth histological examination of brainstem and cerebellum, was performed on 42 stillbirths, aged from 22 to 40 gestational weeks, 12 of which were explained and 30 were unexplained deaths.

In the sudden unexplained stillbirths a variety of morphological and/or biological abnormalities of different structures and nuclei was found, above all the hypoplasia of the parafacial complex, frequently associated with hypoplasia of the arcuate and pre-Bötzinger nuclei, and with thyrosine-hydroxylase immunonegativity in the locus coeruleus. A significant correlation was also observed between the neuropathologic findings and mother's smoking habit.
\end{abstract}

Keywords: Stillbirth, central autonomic nervous system, brainstem, neuropathology, developmental alterations.

\section{INTRODUCTION}

While society displays an increased awareness of sudden infant death syndrome (SIDS), less attention has been focused on another form of unexplained death that occurs with a greater frequency in late pregnancy, namely "stillbirth". In fact, despite the high number of unexplained fetal deaths worldwide, very little research has been devoted to the issue of stillbirths, particularly in the anatomopathologic field.

More frequently than is currently admitted, the lethal event seems to be primarily triggered by a stroke of anatomic "lightning", sufficient to impair the vital functions.

Accordingly, the vital centers of the central autonomic nervous system need to be given a close pathologic investigation, which may seem a simplistic approach to such an extremely complex problem, yet it can enable a structural control of the reflexogenic neural network.

Stillbirth or fetal death is defined as death prior to complete expulsion or extraction of a product of conception, irrespective of the duration of the pregnancy. The perinatal period commences at 22 completed weeks (154 days) of gestation and ends seven completed days after birth [1]. Statistics and research on stillborn infants have been largely neglected [2-5].

Calls for a routine stillbirth autopsy and for a more standardized stillbirth autopsy protocol have been made by the International Stillbirth Alliance (ISA), a recent organization devoted to understanding the causes and prevention of Stillbirth [6].

Herein we illustrate the results of a neuropathologic study on the autonomic central nervous system in 42 fresh stillbirths, 12 of which were explained and 30 were unexplained deaths. Besides the histopathologic examination of the nuclei and structures of the brainstem, cerebellum and upper thoracic spinal cord in serial histological sections, we

*Address correspondence to this author at the Institute of Pathology, University of Milan, Via della Commenda, 19, 20122 Milan, Italy; Tel: +39-0250320800; Fax: +39-02-50320823; E-mail: luigi.matturri@unimi.it applied immunohistochemical methods to analyze the expression of neurotransmitters (namely catecholamines and somatostatin) regulating important physiological functions already from the first weeks of gestation [7-10] and gliosis, a process of astrocytic activation, indicative of a response to central nervous system injury $[11,12]$.

\section{MATERIALS AND METHODOLOGY}

We studied 42 fresh stillbirths, 28 males and 14 females, aged from 22 to 40 gestational weeks. The gestational age peaked in incidence at 36 to 40 weeks (62\%). The weights of victims ranged from 352 to 3500 grams.

Our collected cases were subjected to complete autopsy, including examination of the placental disk, umbilical cord and membranes and an in-depth histological examination of the autonomic nervous system, according to the protocol issued by the "Lino Rossi" Research Center for the study and prevention of the unexpected perinatal death and SIDS of the University of Milan and available on the web site: http://users.unimi.it/ pathol/sids_e.html [13-16].

A diagnosis of "unexplained stillbirth" was established for 30 fetuses who had died suddenly, with no defined cause of death.

In the remaining 12 victims a precise cause of death was diagnosed at autopsy and they were classified as "explained stillbirths" and considered as control cases.

Intrauterine growth retardation, with a weight below the corresponding reference value, was more frequent in the unexplained stillbirth group compared with the control group.

For every case a complete clinical history, particularly referred to mother, was collected. None of the mothers had any significant pathology. None of the mothers used drugs, high doses of caffeine, sedatives or alcohol. With reference to smoking habit, 16 mothers (38\%) declared that they were active smokers (all of more than 3 cigarettes/day and all before becoming pregnant) and 26 (62\%) were non smokers.

Table 1 summarizes the case profiles in this study, with their relative death diagnosis and mother's smoking status. 
Table 1. Case Profiles of the Study

\begin{tabular}{|c|c|c|c|c|c|c|c|c|c|}
\hline \multirow{2}{*}{ Death Diagnosis } & \multicolumn{2}{|c|}{ Sex } & \multicolumn{2}{|c|}{ Gestational Weeks } & \multicolumn{2}{|c|}{ Weight (g) } & SGA & \multicolumn{2}{|c|}{$\begin{array}{c}\text { Maternal Smoking Habit } \\
\mathbf{n}(\%) \\
\end{array}$} \\
\hline & \multicolumn{2}{|c|}{$\mathrm{n}(\%)$} & & & & & n $(\%)$ & Smokers & Non Smokers \\
\hline unexplained stillbirth $(\mathrm{n}=30)$ & $13(31)$ & $17(40)$ & $25-40$ & $34.5 \pm 5.2$ & $830-3930$ & $2100 \pm 99$ & $26(62)$ & $15(36)$ & $15(36)$ \\
\hline explained stillbirth* $(\mathrm{n}=12)$ & $7(17)$ & $5(12)$ & $22-40$ & $33.4 \pm 5.3$ & $352-3940$ & $2550 \pm 128$ & $4(9)$ & $2(5)$ & $10(24)$ \\
\hline
\end{tabular}

*The death causes were: placental pathologies (4 cases); congenital heart diseases: dilated cardiomyopathy (1 case), Ebstein's anomaly (2 cases); pneumonia (1 case); severe renal dysplasia ( 1 case); septicemia ( 1 case); Down's syndrome (1 case); Potter's syndrome (1 case).

$\mathrm{SGA}=$ small for gestational age

Multiple samples of all organs were fixed in $10 \%$ phosphate-buffered formalin, processed and embedded in paraffin. Sections of $4 \mu \mathrm{m}$ were stained with hematoxylin-eosin and Heidenhaim-azan.

Target of this study was the in-depth histological examination of the central autonomic nervous system. The vital centers, where afferents from peripheral receptors converge on neural circuits processing the efferent reflexogenic responses, are located in the brainstem, linked with the cerebellum and the sympathetic intermediolateral nucleus in the thoracic (T1-T5) cord.

After fixation in $10 \%$ phosphate-buffered formalin, the brainstem, cerebellum and upper spinal cord were processed and embedded in paraffin [12-14]. Transverse serial sections were made at intervals of $30 \mu \mathrm{m}$. For each level, twelve 5 $\mu \mathrm{m}$ sections were obtained, two of which were routinely stained for histological examination using alternately hematoxylin-eosin and Klüver-Barrera stains. Three additional sections at each level were subjected to immunohistochemistry for the study of a) the neurotransmitter somatostatin; b) tyrosine-hydroxylase (TH), an enzyme involved in catecholamine (adrenalin, noradrenalin and dopamine) synthesis and c) gliosis. The remaining sections were saved and stained as deemed necessary for further investigations.

As regards the brainstem, identification in serial sections and analysis were made of the main nuclei, namely the locus coeruleus and the parabrachial/Kölliker-Fuse complex in the pons and mesencephalon, the hypoglossus, the dorsal motor vagal, the tractus solitarius, the ambiguus, the pre-Bötzinger, the inferior olivary and the arcuate nuclei in the medulla oblongata. In the upper thoracic spinal cord the intermediolateral nucleus was examined. Fig. (1) shows a diagram of histological sections of the brainstem indicating the localization of the main nuclei and structures examined.

As regards the cerebellum, the cortex layers (external granular layer, molecular layer, Purkinje cell layer and internal granular layer) and the medullary deep nuclei (the dentate nucleus, the fastigial nucleus, the globose nucleus and the emboliform nucleus) were outlined and analyzed.

Particular care was also paid to the lung examination, especially to the stage of development, evaluated on the basis of the macroscopic criterion used at autopsy, namely the correlation between lung weight and body weight (LW/BW), and of the microscopic criteria, namely the presence of cartilaginous bronchi up to the distal peripheral level and the radial alveolar count (RAC). Assessment of this parameter involves examining at least 10 fields for each case (with a 40x lens) in order to estimate the number of alveoli transected by a perpendicular line drawn from the center of the most peripheral bronchiole (recognizable by not being completely covered by epithelium) to the pleura or the nearest interlobular septum. To carry out this examination, we used samples of fetal lung sectioned parallel to the frontal plane and passing through the hilus. The normal reference values for the last three months of gestation correspond to $\geq 0.022$ for LW/BW and range from 2.2 to 4.4 [17] for the RAC.

\section{Immunohistochemical Methods}

In order to analyze the immunoexpression of somatostatin and tyrosine-hydroxylase and to reveal the presence of reactive astrocytes by the glial fibrillary acidic protein (GFAP) method, we used specific primary antibodies after the application of the avidin-biotin-peroxidase technique, in conformity with conventional immunohistochemical procedures.

A detailed description of the immunohistochemical methods, including the immunopositivity evaluation, applied in this study is available in our previous works $[9,10,18]$.

\section{Morphometric Evaluation}

The morphologic examination of the central autonomic nervous system was supplemented by the morphometric analysis of the main brainstem nuclei, performed with an image analyzer. The following parameters were evaluated and indicated as mean values and standard deviation: nucleus area (expressed in $\mathrm{mm}^{2}$ ), neuronal density (expressed as number of neurons per $\mathrm{mm}^{2}$ ) and neuronal size (cell body area, expressed in $\mu \mathrm{m}^{2}$ ). The in-depth description of the methodology and of the related results can be found in our previous works $[10,19,20]$.

\section{Statistical Analysis}

The statistical significance of direct comparison between the two groups (late unexplained and explained stillbirths) of victims was determined using analysis of variance (ANOVA). The associations between maternal cigarette smoking habit, sudden intrauterine death, and the neuropathological findings were determined using Fisher's exact test. The selected threshold level for statistical significance was $\mathrm{p}<0.05$.

\section{RESULTS}

The histological and immunohistochemical examinations of the brainstem, cerebellum and spinal cord showed frequent morphological and/or biological alterations of various structures and nuclei (Table 2). 


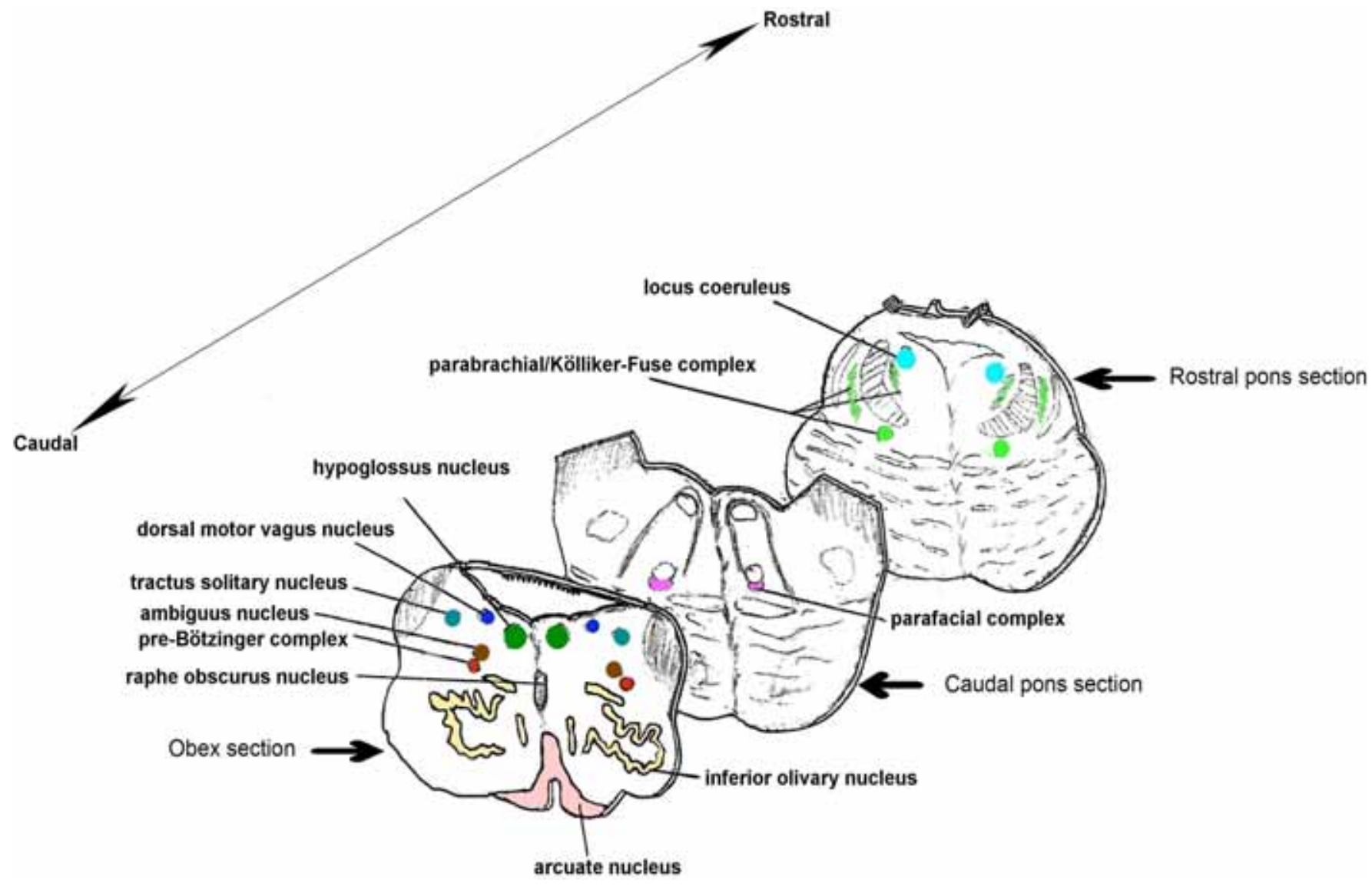

Fig. (1). Diagram of the main histological sections obtained from the brainstem for the anatomo-pathologic examination.

Table 2. Distribution of the Neuropathological Findings in 42 Stillbirths

\begin{tabular}{|c|c|c|}
\hline & Unexplained Stillbirth n (\%) & Explained Stillbirth n (\%) \\
\hline \multicolumn{3}{|l|}{ BRAINSTEM } \\
\hline \multicolumn{3}{|l|}{ Morphological Alterations } \\
\hline PFc hypoplasia & $21(70)^{* *}$ & - \\
\hline ArcN hypoplasia & $17(40)^{* *}$ & $2(5)$ \\
\hline pBN hypoplasia & $13(31)^{* *}$ & - \\
\hline PB/KFc hypoplasia & $8(19)^{*}$ & - \\
\hline \multicolumn{3}{|l|}{ Functional Alterations } \\
\hline SS neg. in HypoglN & $9(21)$ & $2(5)$ \\
\hline $\mathrm{TH}$ neg. in LC & $15(36)^{*}$ & $1(2)$ \\
\hline Brainstem gliosis & $8(19)$ & $2(5)$ \\
\hline \multicolumn{3}{|l|}{ CEREBELLUM } \\
\hline \multicolumn{3}{|l|}{ Morphological Alterations } \\
\hline Cortex immaturity & $10(24)$ & - \\
\hline \multicolumn{3}{|l|}{ Functional Alterations } \\
\hline SS neg. in cortex & $8(19)$ & - \\
\hline DN gliosis & $8(19)$ & $2(5)$ \\
\hline
\end{tabular}

ArcN = arcuate nucleus; DN = dentate nucleus; HypoglN = hypoglossus nucleus; LC = locus coeruleus; $\mathrm{pBN}$ = pre-Bötzinger nucleus; PB/KFc = parabrachial/ Kölliker-Fuse complex $; \mathrm{PFc}=$ parafacial complex; $\mathrm{SS}=$ somatostatin $; \mathrm{TH}=$ thyrosine-hydroxylase .

$* * \mathrm{P}<0.01$ vs explained stillbirths - * $\mathrm{P}<0.05 v s$ explained stillbirths. 


\section{Morphological Alterations}

\section{Brainstem}

Developmental alterations of the arcuate nucleus emerged in 17 of the 30 sudden death victims and in 2 of the 12 stillbirths who had died of known causes. Different degrees of hypoplasia of the arcuate nucleus were observed: bilateral hypoplasia in 6 cases (Fig. 2), partial hypoplasia, generally confined to the inferior two third, in 7 cases, unilateral hypoplasia in 2 cases and complete agenesis in 2 cases.

Hypoplasia of the pre-Bötzinger nucleus, with a decreased number of neuronal bodies and/or dendritic fibers, was diagnosed in 13 victims belonging to the unexplained stillbirth group. Frequently, neuronal cell bodies of the hypoplasic pre-Bötzinger nucleus were small and lengthened with a flattened nucleus, compact chromatin and a poorly evident nucleolus (Fig. 3).

\section{Pons-Mesencephalon}

In transverse sections of rostral pons and caudal mesencephalon of 8 unexplained stillbirth cases but no control cases, a few immature neurons were observed in both the medial and lateral parabrachial nuclei and in the KöllikerFuse area; altogether, these findings led to a diagnosis of hypoplasia of the parabrachial/Kölliker-Fuse complex (Fig. 4). Besides, in caudal pontine sections of 21 victims of unexpected death, we found severe hypoplasia of the parafacial complex.

\section{Cerebellum}

At histological examination, the cerebellar cortex showed an immature structure, uniformly made up of small round

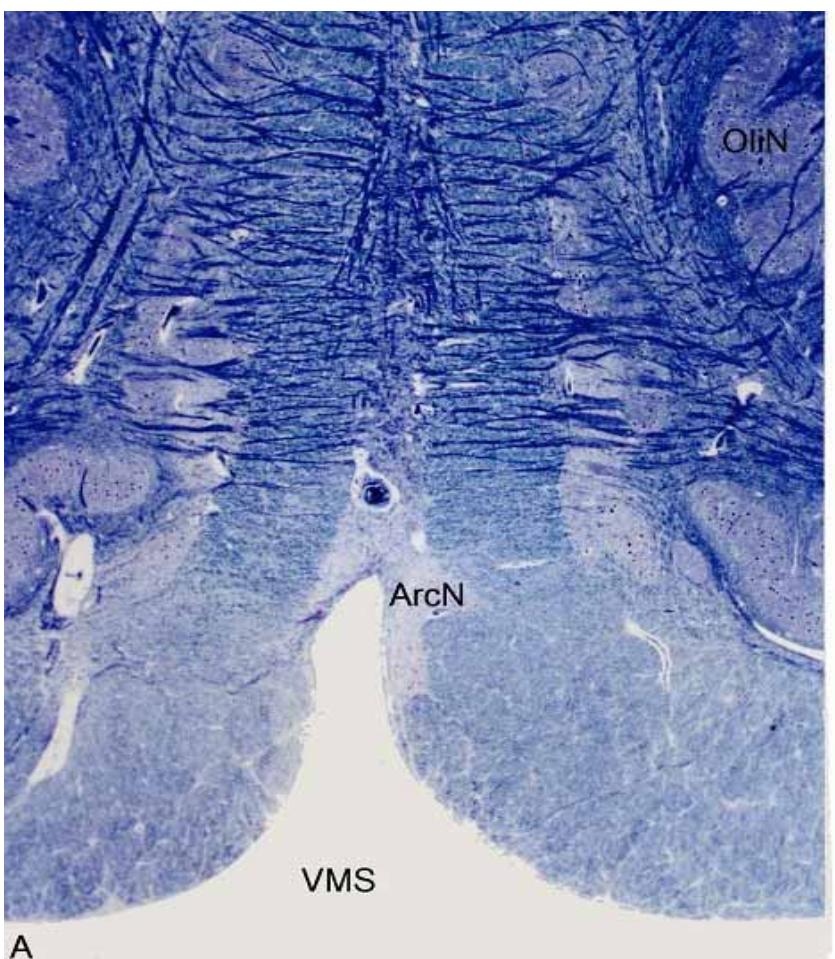

cells without the usual four-layered shape, in 10 unexplained stillbirth cases, aged 35-40 gestational weeks, and in 2 subjects who died at 34 and 40 gestational weeks, respectively, due to placental pathologies.

\section{Functional Alterations}

\section{Brainstem}

We observed negative immunohistochemical expression of somatostatin (a neurotransmitter that usually shows diffuse expression in brainstem nuclei during fetal life) in the hypoglossus nucleus of 9 unexplained stillbirth victims and in 2 control cases, both of whom died at 34 gestational weeks.

\section{Pons-Mesencephalon}

We found a negative or low expression of tyrosinehydroxylase $(\mathrm{TH})$ in the locus coeruleus, a well-known noradrenergic center involved in catecholamine synthesis, in 15 sudden unexplained deaths and in only 1 control case that died of severe chorioamnionitis.

\section{Cerebellum}

There was immunonegativity for somatostatin, that is usually widespread in all the cortex layers in prenatal life, in the Purkinje cells of 8 unexplained stillbirth victims.

A diffuse gliosis was identified by the GFAP method in the brainstem and cerebellum of 8 sudden fetal death victims and in 2 subjects of the control group. In particular, a significant higher number of reactive astrocytes was visible in these cases in the region of the tractus solitary nucleus and of the dentate nucleus.

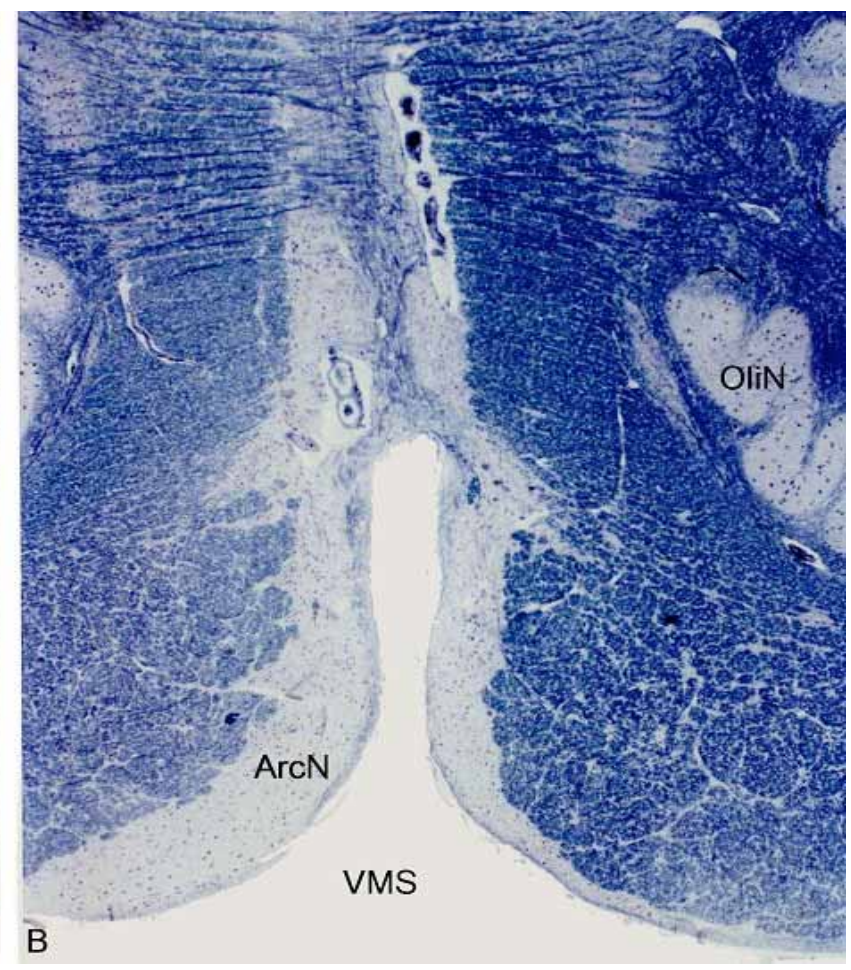

Fig. (2). A) Bilateral hypoplasia of the arcuate nucleus in a case of sudden intrauterine unexplained death at 34 gestational weeks, compared with a normal arcuate nucleus, B) in a fetus died at the same gestational week. Klüver-Barrera stain; Magnification: 25x. ArcN: arcuate nucleus; OliN: inferior olivary nucleus; VMS: ventral medullary surface. 

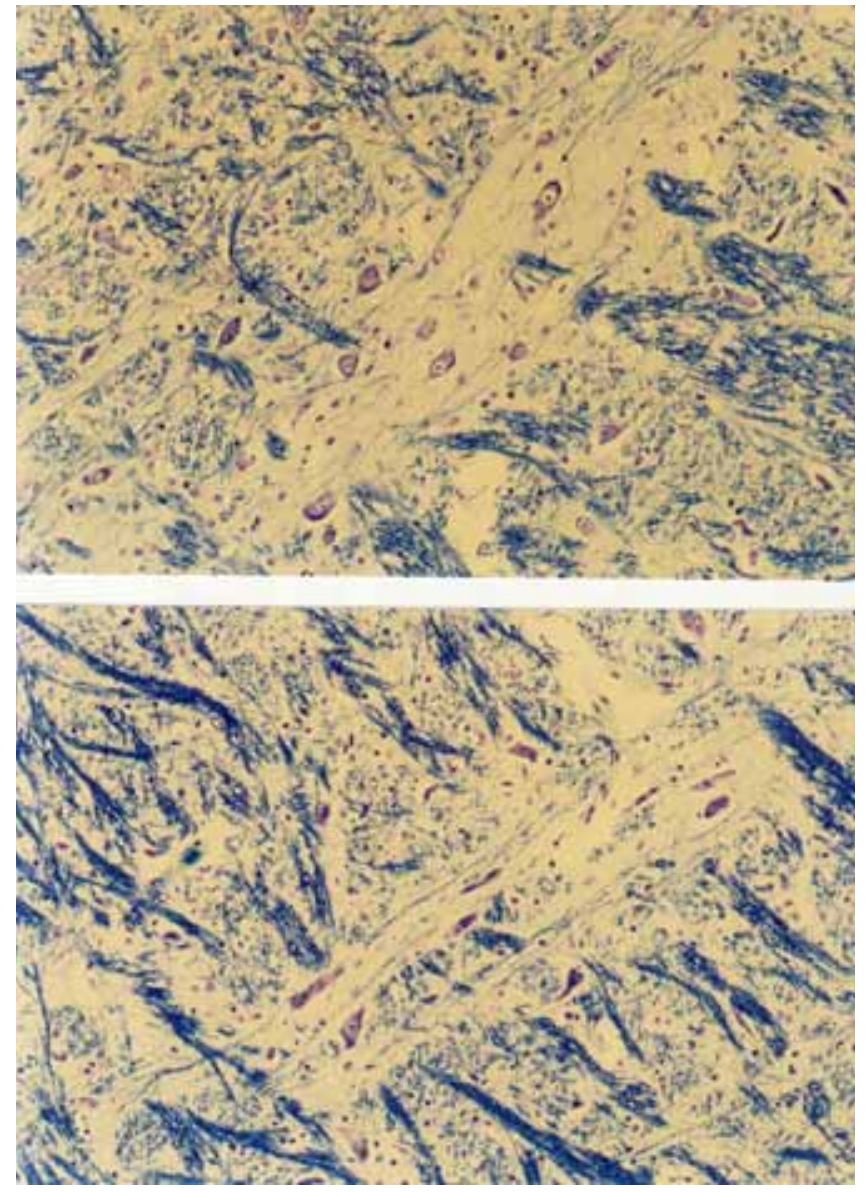

Fig. (3). In the upper image: Normal pre-Bötzinger complex configuration. In the image below: Hypoplasia of the pre-Bötzinger complex with flattened neuronal cell bodies in a case of sudden intrauterine unexplained death at 36 gestational weeks. KlüverBarrera stain; Magnification: 40x.

Histological and immunohistochemical examination of the sympathetic center in the intermediolateral column of the T3-T4 spinal cord, disclosed no morphological and/or functional anomalies.

Frequently, two or more alterations were observable in the same case. In the unexplained stillbirth victims there was a high statistically significant association of hypoplasia of the parafacial complex with hypoplasia of the arcuate nucleus and of the pre-Bötzinger nucleus as well as of decreased TH synthesis in the locus coeruleus $(\mathrm{p}<0.01)$.

Finally, we correlated the two stillbirth groups and the neurodevelopmental defects of the central autonomic nervous system with the mother's smoking habit. We observed a significant correlation between maternal smoking and the following parameters: unexplained stillbirth, hypoplasia of the parafacial complex, hypoplasia of the arcuate nucleus, hypoplasia of the pre-Bötzinger nucleus, hypoplasia of the parabrachial/Kölliker-Fuse complex, and TH negativity in the locus coeruleus (Table $\mathbf{3}$ ).

Histological examination of the other organ samples did not reveal pathologic processes in the unexplained death group.

Bilateral pulmonary hypoplasia was identified in 11 cases $(26 \%)$, characterized by a decreased volume and/or weight of the lungs, altered lung development indices, with a LW/BW value below 0.022 , a RAC index below 2.2 and the presence of cartilaginous bronchi up to the distal peripheral level. In 4 cases, lung hypoplasia was associated with hypoplasia of the parafacial complex and of the arcuate nucleus and parabrachial/Kölliker-Fuse complex and in 2 cases only with hypoplasia of the arcuate nucleus and of the pre-Bötzinger nucleus.
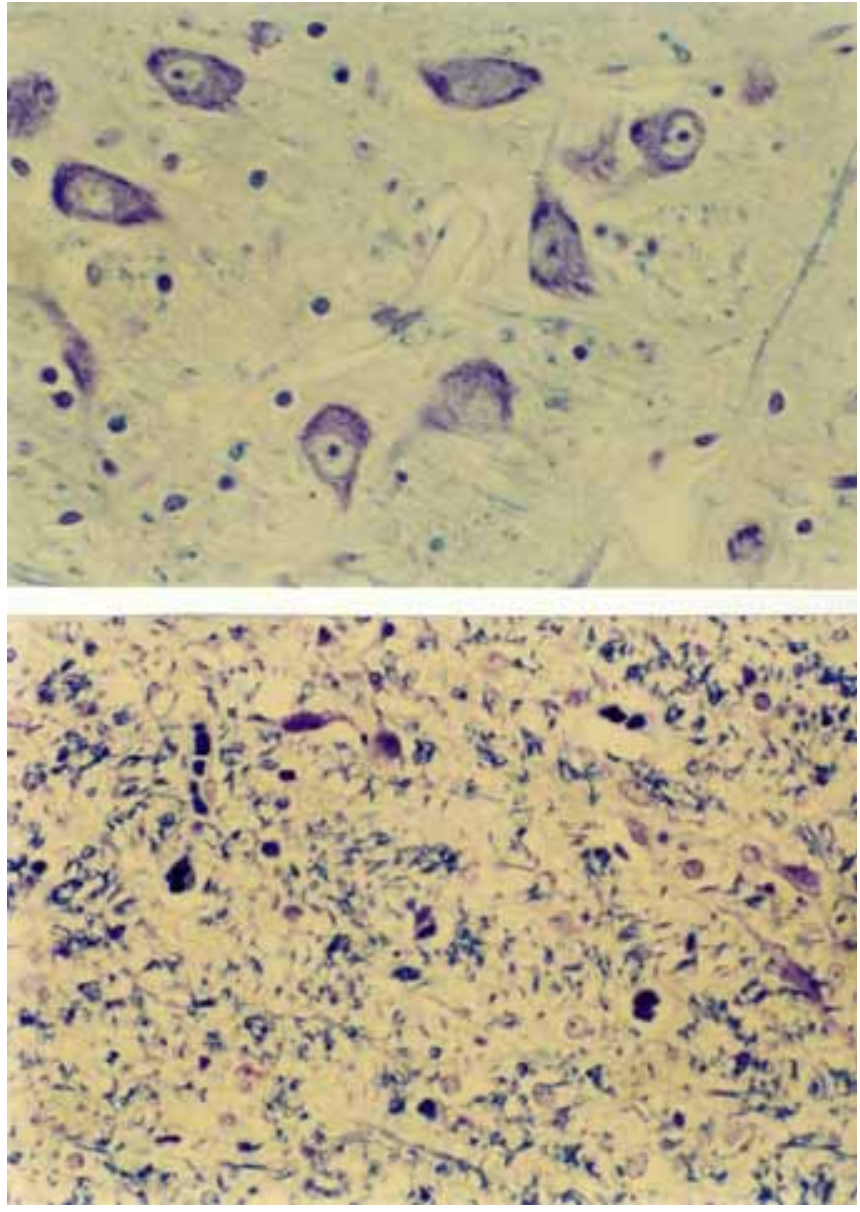

Fig. (4). In the upper image: Normal Kölliker-Fuse complex configuration. In the image below: Hypoplasia of the Kölliker-Fuse complex with absence of the typical large neurons in a case of sudden intrauterine unexplained death at 40 gestational weeks. KlüverBarrera stain; Magnification: 40x.

\section{DISCUSSION}

The World Health Organization (WHO) estimates that 4.5 million stillbirths occur each year worldwide [1]. In the western world one in 100-200 pregnancies ends in fetal death; in addition, late unexplained stillbirth is the largest single cause of death. In some studies more than $60-80 \%$ of stillbirths are unexplained, mainly due to failure to carry out a complete, appropriate post-mortem investigation.

The importance of a thorough autopsy, in cases of fresh stillbirth, to individuate the morphological substrate of death, has been stressed [2,21-24]. Nevertheless, the International Stillbirth Alliance (ISA) underlines that there is no standard method of performing autopsies and that guidelines for the neuropathologic investigation of stillbirth are needed [6]. 
Table 3. Distribution of Neuropathological Findings in Unexplained and Explained Stillbirths in Relation to Maternal Smoking Habit

\begin{tabular}{|c|c|c|c|c|}
\hline \multirow{2}{*}{$\begin{array}{l}\text { Alterations of the Central } \\
\text { Autonomic Nervous System }\end{array}$} & \multicolumn{2}{|c|}{ Smoker Mothers } & \multicolumn{2}{|c|}{ Non-Smoker Mothers } \\
\hline & $\begin{array}{c}\text { Unexplained Stillbirth } \\
\text { n (\%) }\end{array}$ & $\begin{array}{c}\text { Explained Stillbirth } \\
\text { n(\%) }\end{array}$ & $\begin{array}{c}\text { Unexplained Stillbirth } \\
\mathrm{n}(\%)\end{array}$ & $\begin{array}{c}\text { Explained Stillbirth } \\
\mathbf{n}(\%)\end{array}$ \\
\hline ArcN hypoplasia & $15(36)^{* *}$ & $1(2)$ & $2(5)$ & $1(2)$ \\
\hline pBN hypoplasia & $10(24)^{* *}$ & $1(2)$ & $2(5)$ & - \\
\hline $\mathrm{PB} / \mathrm{KFc}$ hypoplasia & $5(12)^{*}$ & $1(2)$ & $1(2)$ & $1(2)$ \\
\hline PFc hypoplasia & $18(50)^{* *}$ & - & $2(5)$ & $1(2)$ \\
\hline SS negativity in HypoglN & $6(14)$ & $2(5)$ & $3(7)$ & $1(2)$ \\
\hline SS negativity in CC & $5(12)$ & - & $3(7)$ & - \\
\hline Brainstem gliosis & $8(19)$ & $2(5)$ & - & - \\
\hline $\mathrm{TH}$ negativity in LC & $14(33)^{* *}$ & $1(2)$ & $1(2)$ & - \\
\hline CC immaturity & $7(17)$ & $2(5)$ & $3(7)$ & - \\
\hline DN gliosis & $8(19)$ & $2(5)$ & - & - \\
\hline
\end{tabular}

ArcN = arcuate nucleus; $\mathrm{CC}=$ cerebellar cortex $\mathrm{DN}=$ dentate nucleus; $\mathrm{Hypog} \mathrm{lN}=$ hypoglossus nucleus; $\mathrm{LC}=$ locus coeruleus; $\mathrm{pBN}=$ pre-Bötzinger nucleus; $\mathrm{PB} / \mathrm{KFc}=$ parabrachial/ Kölliker-Fuse complex; $\mathrm{PFc}=$ parafacial complex; $\mathrm{SS}=$ somatostatin; $\mathrm{TH}=$ thyrosine-hydroxylase

Significance related to controls (explained stillbirths) are expressed for $* \mathrm{P}<0.05$, $* * \mathrm{P}<0.01$.

This situation should improve in Italy. In fact, the Lombardy Region has established the mandatory performance of an autopsy examination in all stillbirths after the $22^{\text {nd }}$ week of gestation, on the basis of the recently approved Italian law [25].

The present study on stillbirth was focused on the central autonomic nervous system, encompassing the brainstem, the cerebellum and the upper thoracic spinal cord, in 42 fresh stillbirths, 28 males and 14 females, aged from 22 to 40 gestational weeks. For 30 fetuses, 17 females and 13 males, with a mean gestational age of 35 weeks, a diagnosis of "unexplained stillbirth" was established. In the remaining 12 victims, 5 females and 7 males, with a mean age of 34 gestational weeks, a precise cause of death was instead diagnosed at autopsy ("explained stillbirth").

We found, particularly in the unexplained stillbirth group, a variety of congenital abnormalities of the central autonomic nervous system. Among the morphological alterations, hypoplasia of the parafacial complex, the master generator of the respiratory rhythm in mammals, and hypoplasia of the arcuate nucleus, a chemoreceptorial component of the ventral medullary surface, were frequent. These alterations were detected respectively in $21(70 \%)$ and in $17(57 \%)$ of the 30 unexplained stillbirths.

All the nuclei and structures that showed alterations in this study, are notoriously involved in respiratory control.

In particular, the parafacial complex, the pre-Bötzinger nucleus and the parabrachial/Kölliker-Fuse complex, that have been well described in experimental studies [26-29] but only recently characterized in human brainstem $[19,30]$, play an important role in breathing control. The parafacial and pre-Bötzinger nuclei are involved in generating the respiratory rhythm. In addition, the ponto-mesencephalic KöllikerFuse nucleus has a preeminent function during intrauterine life, inhibiting the response of central and peripheral chemoreceptors (which are already fully formed and poten- tially functional), and therefore any respiratory reflex. From birth, the inhibitory effects of this nucleus abruptly decline and it becomes active as a respiratory center able to coordinate the pulmonary motor responses to blood oscillations of $\mathrm{pO}_{2}, \mathrm{pCO}_{2}$ and $\mathrm{pH}[18,31,32]$.

Even the cerebellar cortex is a putative neuronal region subserving ventilatory control, since the axons of the granule and Purkinje cells seem to have connections with dorsal and ventral medulla and with specific areas in the pons, classically implicated in respiratory control. Besides, the cerebellar cortex contributes to the control of the respiratory muscles that are involved in restoring blood pressure and breathing rhythm in hypoxic conditions [33,34].

Likewise, the functional alterations observed in this study, consisting of the lack of synthesis of important neurotransmitters in specific nuclei and structures, namely noradrenalin in the locus coeruleus and somatostatin in the hypoglossus nucleus and cerebellar cortex, may interfere with the respiratory mechanism $[9,10,33,34]$.

Although a functional respiratory network is necessary for survival at birth, respiratory-like movements are detectable before birth not only in experimental animals $[35,36]$ but also in humans [17,37]. Thus, an immature neuronal respiratory network is usually active at low frequency in prenatal stages, shortly after the onset of fetal movements. Consequently, the observed morpho-functional alterations of the central autonomic nervous system could determine defects of this occasional respiratory activity in prenatal life. This is borne out by the frequent finding of lung hypoplasia in sudden stillbirth.

Nevertheless, these alterations would not be sufficient to justify fetal death. One possibility is that the neurons of the structures involved not only participate in respiratory modulation but, more extensively, are essential to the control of all vital functions. 
Advancing towards the time of birth, very likely a general check of all the essential functions for extra-uterine life occurs. Sudden unexpected fetal death could therefore be ascribed to a selective process in presence of developmental alterations of the central autonomic nervous system.

Astrocytic gliosis, that we have identified by immunoreactivity to the glial fibrillary acidic protein (GFAP) in the brainstem and cerebellum, is a non-specific secondary response to brain injuries $[11,12]$. In particular, hypoxic events can induce the proliferation of activated astrocytes in areas that are important in the physiology of breathing.

The possible risk factors for hypoxic events in the fetal period include maternal smoking [20,38-40] and likely air pollution [41]. In cases of maternal smoking in pregnancy, carbon monoxide, a gaseous combustion product of nicotine, may readily spread by passive diffusion into the placenta, where it binds to hemoglobin. Consequently, carboxyhemoglobin, that generally has $15 \%$ higher concentrations in the fetal compartment than the maternal levels [42], inhibits the release of oxygen, causing altered physiological development in the fetal organs and tissues, especially those most susceptible to hypoxic damage, including the brain $[43,44]$. Besides, nicotine is one of the few lipid-soluble substances that, by crossing the blood-brain barrier, can act directly on the expression of genes that control the developing nervous system [45]. In addition, air pollution, particularly in the Lombardy Region where the victims of this study come from, and which features high rates of both gases (carbon monoxide, nitrogen dioxide, ozone, sulphur dioxide) and particulate matter (above all $\mathrm{PM}_{10}$, with a median diameter inferior to $10 \mu \mathrm{m}$ ) [41], could have an important influence in determining sudden fetal death. We postulate that gas pollutants in particular, likewise cigarette smoke, can cross the placenta during pregnancy in the maternal blood, and lead to structural and/or functional impairments of the central nervous system.

Smoking has multiple effects and consequences. Apart from the overall toxic effect and likely induction of genetic alterations, it also causes atherosclerotic alterations [46,47], whose consequences contribute both to tissue hypoxia and to altered development of the autonomic nervous system.

Gliosis, although observed in only a small percentage of unexplained stillbirths, is likely an expression of the tissue reaction to cell necrosis caused by hypoxemia. The study by Grafe and Kinney supports this view [48]. These authors found a variety of abnormalities in the brains of stillborns, the most common including cerebral white matter gliosis, related to hypoxia/ischemia in the perinatal period.

\section{CONCLUSIONS}

The present neuropathologic study has contributed to identify the nature and frequency of alterations of the central autonomic nervous system in stillbirth, particularly in unexplained stillbirth. These alterations are to a large extent congenital, being an expression of disturbed development in pathophysiologic response to a variety of mechanisms and above all to maternal cigarette smoking in pregnancy and/or to air pollution.

The well known peculiar reactivity and sensitivity of the fetal tissues causes the spread of both primary and secondary (gliosis) reactive alterations of both the brainstem and the entire encephalon. Inevitably, impairment of the brainstem, the site of convergence of vagal-glossal-pharyngeal stimuli, plays a major role in causing death. Therefore, in stillbirth an in-depth pathologic examination of the autonomic nervous system, combined with a careful survey of the individual case history, is very important.

The recent recognition of a common pathologic substrate in both unexplained stillbirth and SIDS [49], consisting of congenital anomalies of the nuclei and/or structures of the autonomic nervous system that preside over the vital activities, suggests that appropriate anatomopathologic research in the autonomic nervous system field may not only explain the pathogenesis of unexplained stillbirth but also individuate the morphological substrates of infant sudden death.

However, it is extremely important that such a thorough autopsy of fresh stillbirths be performed only by experienced, reliable pathologists, as stipulated at the 7th International Conference on SIDS in 2002 [50], and established by Italian law [25].

\section{ACKNOWLEDGEMENTS}

This study was supported by the Italian Lombardy Region target project n. 49210/2000 (Program of research and intervention for the reduction of the risk of SIDS and unexpected fetal death), by the agreement Lombardy RegionPfizer Italia S.r.l. n. 814/2006, and by Ministry of Foreign Affairs (joined projects of particular relevance "Anatomopathologic and genetic study of the unexplained perinatal death and SIDS") n. 269/P/0085087/2004 and n. 0083227/20006.

\section{ABBREVIATIONS}

\begin{tabular}{|c|c|c|}
\hline $\mathrm{ArcN}$ & $=$ & Arcuate nucleus \\
\hline $\mathrm{DN}$ & $=$ & Dentate nucleus \\
\hline HypoglN & $=$ & Hypoglossus nucleus \\
\hline $\mathrm{LC}$ & $=$ & Locus coeruleus \\
\hline $\mathrm{pBN}$ & $=$ & Pre-Bötzinger nucleus \\
\hline $\mathrm{PB} / \mathrm{KFc}$ & $=$ & Parabrachial/ Kölliker-Fuse complex \\
\hline $\mathrm{PFc}$ & $=$ & Parafacial complex \\
\hline SS & $=$ & Somatostatin \\
\hline $\mathrm{TH}$ & $=$ & Thyrosine-hydroxilase \\
\hline
\end{tabular}

\section{REFERENCES}

[1] World Health Organization (WHO). Neonatal and perinatal mortality: country, regional and global estimates 2006; 1-75.

[2] Cartlidge PHT, Dawson AT, Stewart JH, Vujanic GM. Value and quality of perinatal and infant postmortem examination: cohort analysis of 400 consecutive deaths. BMJ 1995; 310: 155-58.

[3] Stanton C, Lawn JE, Rahman H, Wilczynska-Ketende K, Hill K. Stillbirth rates: delivering estimates in 190 countries. Lancet 2006; 367: 1487-94.

[4] McClure EM, Nalubamba-Phiri M, Goldenberg RL. Stillbirth in developing countries. Int J Gynaecol Obstet 2006; 94: 82-90.

[5] Magee JF. Investigation of Stillbirth. Ped Dev Pathol 2001; 4: 1-22.

[6] International Stillbirth Alliance. Available at http://www.stillbirthalliance.org/

[7] Bacopoulos NG, Bhatnagar RK. Correlation between tyrosine activity and catecholamine concentration or turnover in brain regions. J Neurochem 1977; 29: 639-43. 
[8] Carpentier V, Vaudry H, Laquerrière A, Tayot J, Leroux P. Distribution of somatostatin receptors in the adult human brainstem. Brain Res 1996; 734: 135-48.

[9] Lavezzi AM, Ottaviani G, Matturri L. Role of somatostatin and apoptosis in breathing control in sudden perinatal and infant unexplained death. Clin Neuropathol 2004; 23: 304-10.

[10] Lavezzi AM, Ottaviani G, Mingrone R, Matturri L. Analysis of the human locus coeruleus in perinatal and infant sudden unexplained death. Possible role of the cigarette smoking in the development of this nucleus. Dev Brain Res 2005; 154: 71-80.

[11] Blinkov SM. Glial index and distribution density of glial cells in the human brainstem. Ark Anat 1963; 45: 42-7.

[12] Colodner KJ, Montana RA, Anthony DC, et al. Proliferative potential of human astrocytes. J Neuropathol Exp Neurol 2005; 64: 1639 .

[13] Matturri L, Ottaviani G, Alfonsi G, Crippa M, Rossi L, Lavezzi AM. Study of the brainstem, particularly the arcuate nucleus, in sudden infant death syndrome (SIDS) and sudden intrauterine unexplained death (SIUD). Am J Forensic Med Pathol 2004; 25: 44-8.

[14] Matturri L, Ottaviani G, Alfonsi G, Rossi L, Lavezzi AM. Guidelines in pathological and forensic-medical diagnostics of sudden unexpected infant (SIDS) and fetal death. Lombardy Region Project for reduction of the risk for SIDS and/or Sudden Fetal Death; 2005; available at: http://users.unimi.it/ pathol/sids/linee_guida_e. html.

[15] Matturri L, Ottaviani G, Lavezzi AM. Techniques and criteria in pathologic and forensic-medical diagnostics of sudden unexpected infant and perinatal death. Am J Clin Pathol 2005; 124: 259-68.

[16] Lombardy Regional Decree n. 11693 of 06-20-2002 "Legislative measures on adoption of anatomo-pathological and forensicpathological intervention targeted on the prevention, knowledge, and identification of the sudden infant death cases".

[17] Matturri L, Minoli I, Lavezzi AM, et al. Hypoplasia of meduallary arcuate nucleus in unexpected late fetal death (stillborn infants): a pathologic study. Pediatrics 2002; 109: E43.

[18] Lavezzi AM, Ottaviani G, Mauri M, Matturri L. Biopathology of the olivocerebellar network in sudden unexplained perinatal and sudden infant death syndrome related to maternal cigarette smoking. Neurol Res 2007; [Epub ahead of print].

[19] Lavezzi AM, Ottaviani G, Rossi L, Matturri L. Cytoarchitectural organization of the parabrachial/Kölliker-Fuse complex in man. Brain Dev 2004; 26: 316-20.

[20] Lavezzi AM, Ottaviani G, Mauri M, Matturri L. Hypoplasia of the arcuate nucleus and maternal smoking during pregnancy, in perinatal and infant sudden unexpected death. Neuropathology 2004; 24 : 284-89.

[21] Hefler LA, Hersh DR, Moore PJ, Gregg A. Clinical value of postnatal autopsy and genetic consultation in fetal death. Am J Med Genet 2001; 104: 165-68.

[22] Incerpi MH, Miller DA, Samadi R, Settlage RH, Goldwin TM. Stillbirth evaluation: what tests are needed? Am J Obstet Gynecol 1998; 178: 1121-25.

[23] Okah FA. The autopsy: experience of a regional neonatal intensive care uniy. Paediatr Perinat Epidemiol 2002; 16: 350-54.

[24] Faye-Petersen OM, Guinn DA, Wenstrom KD. Value of perinatal autopsy. Obstet Gynecol 1999; 94: 915-20.

[25] Italian Law n. 31; "Regulations for Diagnostic Post Mortem Investigation in Victims of Sudden Infant Death Syndrome (SIDS) and Unexpected Fetal Death", 2006.

[26] Rekling JC, Feldman JL. PreBötzinger complex and pacemaker neurons: hypothesized site and kernel for respiratory rhythm generation. Annu Rev Physiol 1998; 60: 385-405.

[27] Chatonnet F, Borday C, Wrobel L, et al. Ontogeny of central rhythm generation in chicks and rodents. Respir Physiol 2006; 154: 37-56.
[28] Guyenet PG. Novel two-rhythm generator theory of breathing in mammals. J Physiol 2006; 570: 407-20.

[29] Chamberlin NL, Saper CB. Topographic organization of respiratory responses to glutamate microstimulation of the parabrachial nucleus in the rat. J Neurosci 1994; 4: 6500-10.

[30] Lavezzi AM, Matturri L. Functional neuroanatomy of the human pre-Bötzinger complex with particular reference to sudden unexplained perinatal and infant death. Neuropathology 2007; [Epub ahead of print].

[31] Cohen M. Neurogenesis of respiratory rhythm in the mammal. Physiol Rev 1979; 59: 91-104.

[32] Fulwiler CF, Saper CB. Subnuclear organization of the efferent connections of the parabrachial nucleus in the rat. Brain Res Rev 1984; $7: 229-59$.

[33] Lavezzi AM, Ottaviani G, Matturri L. Alterations of biological features of the cerebellum in sudden perinatal and infant death. Curr Mol Med 2006; 6: 429-35.

[34] Lavezzi AM, Ottaviani G, Terni L, Matturri L. Histological and biological developmental characterization of the human cerebellar cortex. Int J Dev Neurosci 2006; 24: 365-71.

[35] Bystreycka EB, Nail BS, Purves MJ. Central and peripheral neural respiratory activity in the mature sheep fetus and newborn lamb. Respir Physiol 1975; 25: 199-215.

[36] Dawes GS, Fox HE, Leduc BM, Liggins GC, Richards RT. Respiratory movements and rapid eye movement sleep in the fetal lamb. J Physiol 1972; 220: 119-43.

[37] Blanco CE, Hanson MA, Kumar P. Breathing and sleep states in the fetus and at birth. In: Loughlin GM, Carrol JL, Marcus CL (Eds), Sleep and Breathing in Children. A Developmental Approach. Marcel Dekker, Inc. New York, 1995; 161-79.

[38] Kleinman JC, Pierre MB, Madans JH. The effect of maternal smoking on fetal and infant mortality. Am J Epidemiol 1988; 127: 274 82 .

[39] Hofvendahl EA. Smoking in pregnancy as a risk factor for longterm mortality in the offspring. Paediatr Perinat Epidemiol 1995;9: 381-99.

[40] Cnattingius S, Haglund B, Meirik O. Cigarette smoking as risk factor for late fetal and early neonatal death. BMJ 1988; 297: 25826.

[41] Schweda D. Air pollution and health in urban areas. Rev Environ Health 2000; 15: 13-41.

[42] Lambers DS, Clark K. The maternal and fetal physiologic effects of nicotine. Semin Perinatol 1996; 20: 115-26.

[43] Gressens P, Laudenbach V, Marret S. Mechanisms of action of tobacco smoke on the developing brain. J Gynecol Obstet Biol Reprod (Paris) 2003; 32 (1 Suppl): 30-2.

[44] Gospe, SM, Zhou SS, Pinkerton KE. Effects of environmental tobacco smoke exposure in utero on brain development. Pediatr Res 1996; 39: 494-8.

[45] Cutler RWP, Sperrel RB. Cerebrospinal fluid: a selective review. Ann Neurol 1982; 11: 1-16.

[46] Matturri L, Lavezzi AM, Ottaviani G, Rossi L. Intimal preatherosclerotic thickening of the coronary arteries in human fetuses of smoker mothers. J Thromb Haemost 2003; 1: 2234-38.

[47] Matturri L, Ottaviani G, Lavezzi AM. Early atherosclerosis lesions in infancy: role of parental cigarette smoking. Virchows Arch 2005; 447: 74-80.

[48] Grafe MR, Kinney HC. Neuropathology associated with stillbirth. Semin Perinatol 2002; 26: 83-8.

[49] Matturri L, Ottaviani G, Nonnis Marzano F, Lavezzi AM. Stillbirth versus SIDS. Pathology of the autonomic nervous system and DNA polymorphisms in SIUD and SIDS. 2006; Proceed. $9^{\text {th }}$ SIDS International Conference, Yokohama, Japan 2006, pp.105.

[50] Matturri L, Lavezzi AM, Rossi L. Proposal to modify the definition of SIDS, with regard to the post mortem exam. Proceed. $7^{\text {th }}$ SIDS International Conference. Florence, Italy 2002; pp. 103. 